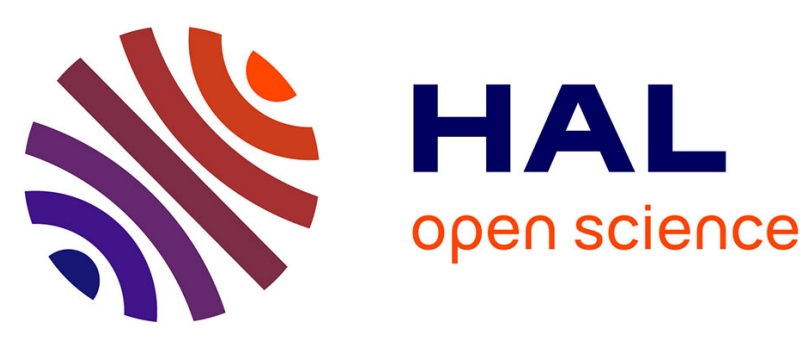

\title{
Numerical and Experimental Investigation on Contactless Resonant Sensors
}

\author{
Carlo Trigona, Salvatore Baglio, Bruno Andò, Nicolò Savalli, Marco Baù, \\ Vittorio Ferrari, Mauro Serpelloni, Emilio Sardini
}

\section{- To cite this version:}

Carlo Trigona, Salvatore Baglio, Bruno Andò, Nicolò Savalli, Marco Baù, et al.. Numerical and Experimental Investigation on Contactless Resonant Sensors. Sensors and Actuators A: Physical , 2010, 162 (2), pp.329-335. 10.1016/j.sna.2010.04.009 . lirmm-00580564

\section{HAL Id: lirmm-00580564 https://hal-lirmm.ccsd.cnrs.fr/lirmm-00580564}

Submitted on 28 Mar 2011

HAL is a multi-disciplinary open access archive for the deposit and dissemination of scientific research documents, whether they are published or not. The documents may come from teaching and research institutions in France or abroad, or from public or private research centers.
L'archive ouverte pluridisciplinaire HAL, est destinée au dépôt et à la diffusion de documents scientifiques de niveau recherche, publiés ou non, émanant des établissements d'enseignement et de recherche français ou étrangers, des laboratoires publics ou privés. 


\title{
Numerical and experimental investigation on contactless resonant sensors
}

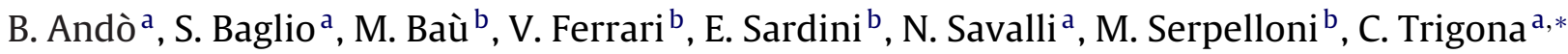 \\ a Dipartimento di Ingegneria Elettrica Elettronica e dei Sistemi, D.I.E.E.S. University of Catania, Viale Andrea Doria 6, 95125, Catania, Italy \\ b Dipartimento di Elettronica per l'Automazione, University of Brescia, via Branze 38, I25123, Brescia, Italy
}

\section{A R T I C L E I N F O}

\section{Article history:}

Received 30 September 2009

Received in revised form 8 February 2010

Accepted 5 April 2010

Available online 31 May 2010

\section{Keywords:}

Contactless MEMS

Crab-leg microresonator

BESOI technology

Numerical and experimental investigation

\begin{abstract}
A B S T R A C T
This paper reports numerical and experimental investigation on a (bulk and etch silicon on insulator) BESOI MEMS device. The implemented contactless actuation principle, exploits Lorentz forces exerted on a conductive-non magnetic surface of the sensor. These forces derive from the interaction between the eddy-currents and the radial magnetic field, both generated by a sinusoidally driven external inductor. Both excitation and readout strategies are performed remotely via a magnetic strategy. The sensor proposed here has been first analytically and numerically studied by using CoventorWare ${ }^{\mathrm{TM}} 2008$, then the device prototype has been fabricated and a preliminary experimental campaign has been performed to characterize the system in terms of variation of its resonance frequency against changes in the sensor mass.
\end{abstract}

(c) 2010 Elsevier B.V. All rights reserved.

\section{Introduction}

Resonant sensors that can be remotely interrogated offer the possibility to develop interesting applications, for example, in the monitoring of hermetic areas or protected environments. Moreover, adverse, hazardous and inaccessible places are often incompatible not only with the "wired" solution but also with the presence of active electronics. A possible solution is represented by a contactless readout strategy that can be adopted to measure different physical quantities of interest (viscosity, temperature, pressure, etc.) from a passive sensor located in a risky and unsafe environment. The typical approach to sense a physical quantity is represented by a measurement node which houses both sensors and conditioning circuit. Generally, an electrical connection between the sensing element and the readout circuit is required, which is based on cables or field buses. However, it is often interesting to monitor physical or chemical quantities in specific operating conditions which require the circuitry to be separated from the sensing element. Despite their intrinsic robustness, one of the main drawbacks of cabled solutions is their unsuitability in enclosed, inaccessible and sealed areas. For this reason, wireless systems and communication protocols have been proposed as one possible solution. Alternatively novel "uncabled" measurement systems have been realized where the active sensing element is spatially independent from the external signal conditioner. In this new scenario dedicated strategies for the power supply of the active sensing elements are required to be adopted. At present, available power

\footnotetext{
* Corresponding author. Tel.: +39 0957382301.

E-mail address: carlo.trigona@diees.unict.it (C. Trigona).
}

supply approaches can be based on on-board generators, such as batteries, on energy scavenging solutions or on the energy transfer from the external circuit.

This paper addresses the latter approach, namely the energy transfer from the external circuit. Additionally, the work investigates the possibility of contactless exciting and detecting mechanical resonances in micromachined structures by means of an electromagnetic principle. In this context, the adoption of the resonant principle seems particularly promising because of its intrinsic robustness and independence from the particular sensing technique adopted. The working principle proposed here requires the resonator only to be electrically conductive, avoiding on-board circuitry or any specific magnetic property.

The proposed methodology represents a novel approach suitable for passive microresonators realization, in fact only standard MEMS materials (i.e. metal layer for the conductive sensor plate, and the others as structural materials) are employed, without supplementary highly polluting substances or deposition of magnetic alloys.

We therefore here suggest the use of a sensor element totally passive, consisting of a resonant structure that can be excited and interrogated without contact by using an external electromagnetic field; this is promising for performing measurements of different physical quantities [1-4] especially in environments not compatible with the requirements of active electronics.

Several examples of passive contactless resonators, where a movable inertial mass, fabricated by using different materials such as ferrofluids, amorphous FeSiB, silicon mass, etc. can be contactless excited and detected employing magnetic, vibration-based or optical principles have been presented in the scientific literature [1-3]. In this paper, a MEMS microresonator composed by four crab- 


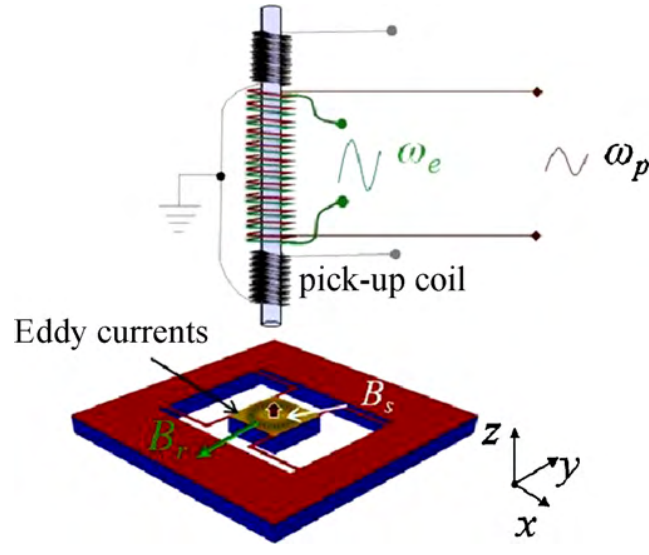

Fig. 1. Schematic diagram of the contactless principle applied to an inertial mass suspended by four silicon beams in a crab-leg configuration. The sinusoidal signal $\omega_{e}$ is used to excite the inertial mass, whereas the bias at pulsation $\omega_{p}$ generates a magnetic field onto the conductive plate (yellow). The output at the pick-up coil is an amplitude modulated signal having a carrier at $\omega_{p}$. (For interpretation of the references to color in this figure legend, the reader is referred to the web version of the article.).

leg beams and a suspended mass has been designed. The device has been fabricated at CNM, Barcelona, Spain by adopting a BESOI (bulk and etch silicon on insulator) micromachining technology. The interrogation contactless working principle, previously studied by the authors [5-7], consists of an actuation principle based on the exploitation of Lorentz forces generated by the interaction between a time-varying magnetic field and the eddy-currents that this magnetic field induces onto a conducting surface. The detection strategy is based on probing the magnetic field induced on the conductive surface of the resonator. The induced magnetic field is modulated by the vibrations of the resonator and it is sensed at a pick-up coil in a differential configuration. Suitable frequency differences are adopted between the actuation and the sensing sections.

The realized device has been numerically and experimentally investigated by means of CoventorWare ${ }^{\mathrm{TM}}$ 2008. The simulator allowed to study the static and dynamic behavior of the micro device and the eddy-currents distribution generated by the inductor. An analytical model dedicated for a crab-leg BESOI structure has been developed and confirmed by a measurement campaign, performed in the MEMS device.

\section{Working principle: a brief overview}

In this section, a brief overview of both the contactless actuation principle and the remote readout strategy is presented; more details can be found in [7-9].

The contactless working principle consists of an actuation principle based on a Lorentz force source and a detection principle realized by a carrier signal and a pick-up coil in a differential configuration.

In Fig. 1 the schematic diagram of the contactless principle applied to a crab-leg microresonator is shown:

- By forcing a periodic bias current at pulsation $\omega_{e}$ in the solenoid a periodic magnetic field is generated. The solenoid is placed such to face the conductive surface of the suspended mass of the resonator, the magnetic field induces therefore an eddy-current density in the conductive plate. The interaction between the eddy-currents and the radial component of the magnetic field $\left(B_{r}\right)$ results into a Lorentz force per unit of volume that moves the conductive mass in the $z$ direction. The force along the $z$ axis $\left(F_{z}\right)$,

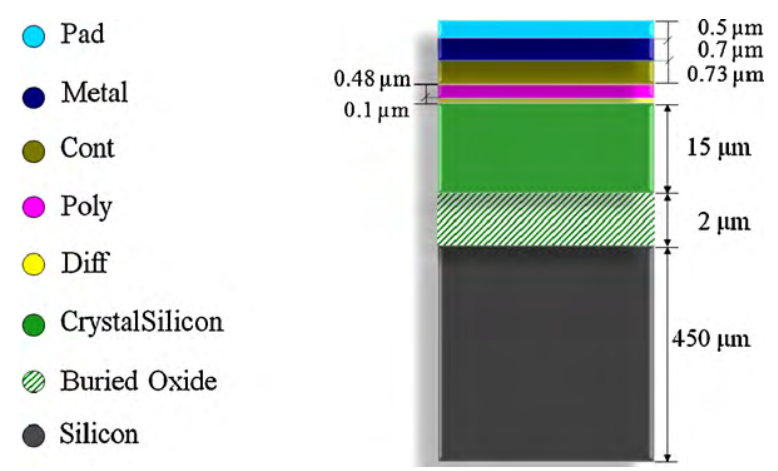

Fig. 2. Cross-sectional area of the bulk and etch silicon on insulator (BESOI) technology used.

can be expressed as follows [7]:

$F_{z}=0.5 \cdot k\left(\omega_{e}\right)\left[B_{r} \cdot \sin (\phi)+B_{r} \cdot \sin \left(2 \omega_{e} t+\phi\right)\right]$

The force expression evinces a constant force component correlated to the conductor plate impedance phase $(\phi)$, and a component that evolves with a pulsation of $2 \omega_{e}$. The force is proportional to the radial component of the magnetic field $B_{r}$ and to the factor $k\left(\omega_{e}\right)$ which depends on the electrical impedance of the conductive surface and on the induced eddy-current density. Adopting an excitation pulsation of $\omega_{e}=\omega_{r} / 2$, where $\omega_{r}$ represents the mechanical resonance pulsation of the device, the system will oscillate at the resonance frequency.

- The detection principle is based on a periodic bias current at pulsation $\omega_{p}$ forced in the solenoid. The induced magnetic field creates an eddy-current density in the conductive plate and generates a magnetic field $B_{s}$. The oscillations of the mass generate an amplitude modulation on the carrier bias and the information can be extracted using the two sensing coils with a frequency domain readout strategy. In the absence of the conductive passive element, only the carrier bias frequency appears; on the contrary, in presence of the resonator two spectral components will appear as consequence of the motion of the structure $\left(\omega_{p} \pm \omega_{r}=\omega_{p} \pm 2 \omega_{e}\right)$. This working principle can be applied to different families of micromachined devices like cantilever beams, bridges, suspended masses with springs, crab-leg structures, etc. In the next section the numerical and experimental investigation of an inertial suspended mass, with four springs in a crab-leg configuration is discussed. This structure offers greater robustness respect to a simple cantilever beam and it is also possible to integrate a consistent inertial mass; this represents an advantage in terms of Lorentz force actuation because a big proof mass implies a low resonance frequency and negligible skin effects.

\section{Investigation on a BESOI crab-leg microresonator}

The MEMS device investigated here is a suspended mass supported by four crab-leg beams, it has been realized by using a BESOI process, available at the Centro Nacional de Microelectronica (CNM) of Barcelona, Spain.

A silicon on insulator (SOI) wafer based on $15 \mu \mathrm{m}$ c-Si layer and $450 \mu \mathrm{m}$ carrier substrate with $2 \mu \mathrm{m}$ of buried oxide, has been processed with a front and back-side DRIE etching technique. Functional materials as metal and polysilicon have been added as shown in Fig. 2. Furthermore, a doping c-silicon procedure based on $\mathrm{POCl}_{3}$ has been used to increase the electrical conductivity.

The process flow can be summarized as follows:

- Doping of c-Silicon, high concentration, about $10^{20}\left(\mathrm{POCl}_{3}\right)$,

$\circ$ thermal grown of oxide on both sides, thickness $=100 \mathrm{~nm}$, at temperature of $1100^{\circ} \mathrm{C}$, 
- deposition of polysilicon on both sides, thickness $=480 \mathrm{~nm}$,

- doping of polysilicon,

- removing the PSG formed during the doping of the polysilicon,

photolithography of polysilicon, mask used: poly,

- RIE etching for removing polysilicon on the front-side, thickness to be removed $=480 \mathrm{~nm}$,

- especial deposition of contact (the oxide between polysilicon and metal) on the front-side, thickness $=130 \mathrm{~nm}$ (no doped oxide), thickness $=600 \mathrm{~nm}$.

- annealing of contact (for reflowing it and planarize the surface of the wafer),

- photolithography of contact, mask used: contact,

- RIE etching of oxides: contact + thermal oxide, thickness to be removed $=600 \mathrm{~nm}+130 \mathrm{~nm}\left(\mathrm{SiO}_{2}\right)+100 \mathrm{~nm}$ (thermal oxide),

o deposition of metal $(\mathrm{Al} / \mathrm{Cu})$ on the front-side, thickness $=700 \mathrm{~nm}$, o photolithography of metal on the front-side, mask used: metal,

- RIE etching for removing metal on the front-side,

- deposition of pad $\left(\mathrm{SiO}_{2}+\mathrm{Si}_{3} \mathrm{~N}_{4}\right)$ on the front-side, thickness $=500 \mathrm{~nm}$,

- removing polysilicon and $\mathrm{SiO}_{2}$ on the back-side,

- deposition of metal $(\mathrm{Al} /-\mathrm{Cu})$ on the back-side, thickness $=1000 \mathrm{~nm}$,

o photolithography of $\mathrm{SiO}_{2}$ on the front-side, mask used: pad.

A silicon suspended mass has been designed with four silicon springs ( $100 \mu \mathrm{m}$ width and a length of $800+1600 \mu \mathrm{m}$ for the segment anchored to the central proof mass and the one anchored to the substrate, respectively).

The physical behavior of the micromachined sensor has been investigated by using CoventorWare ${ }^{\mathrm{TM}} 2008$, and a finite element method (FEM) analysis. Fig. 3a shows the layout of the investigated device, whereas Fig. $3 \mathrm{~b}$ presents the 3D model mesh realized by using an adaptive second order tetrahedrons architecture.

The MemMech tool has been used to analyze the resonance frequency along the $z$ axis (forcing a sinusoidal bias, Fig. 3c) and also the effect of the of the gravity (imposing a constant force value as boundary condition, Fig. 4a).

From the simulation results a resonant frequency of about $500 \mathrm{~Hz}$ has been computed by using the Lanczos solution method. Fig. 4a shows the mass displacement along the easy axis in the absence of supplementary applied load to the plate surface of the microresonator: a maximum value of about $0.3 \mu \mathrm{m}$ has been detect around the center of the conductive plate.

The MemHenry toolbox of CoventorWare ${ }^{\mathrm{TM}}$ has been used to analyze the effect of the eddy-currents induced on the top surface of the device. In order to do this, an external inductor has been considered as variable magnetic source (Fig. 4b).

The device has been also analytically studied by using an energetic approach which takes into the account not only the strain energy density along the beams [8] but also including the contribution of torsion. Furthermore, the model must consider the effect of the materials offered by the BESOI process: for this reason the equivalent section method theory will be included in the analytical study.

The free body analysis of a single crab-leg beam with an applied force along the $z$ axis and by adopting the Castigliano's theorem, the displacement $\left(\delta_{z}\right)$ of the structure as a consequence of a load force $\left(F_{z}\right)$ has been evaluated.

The Hooke's law has been used to derive the elastic constant along the $z$ axis, needful to estimate the resonance frequency [10].

By adopting the heterogeneous beam theory, the spring constant assumes the following form:
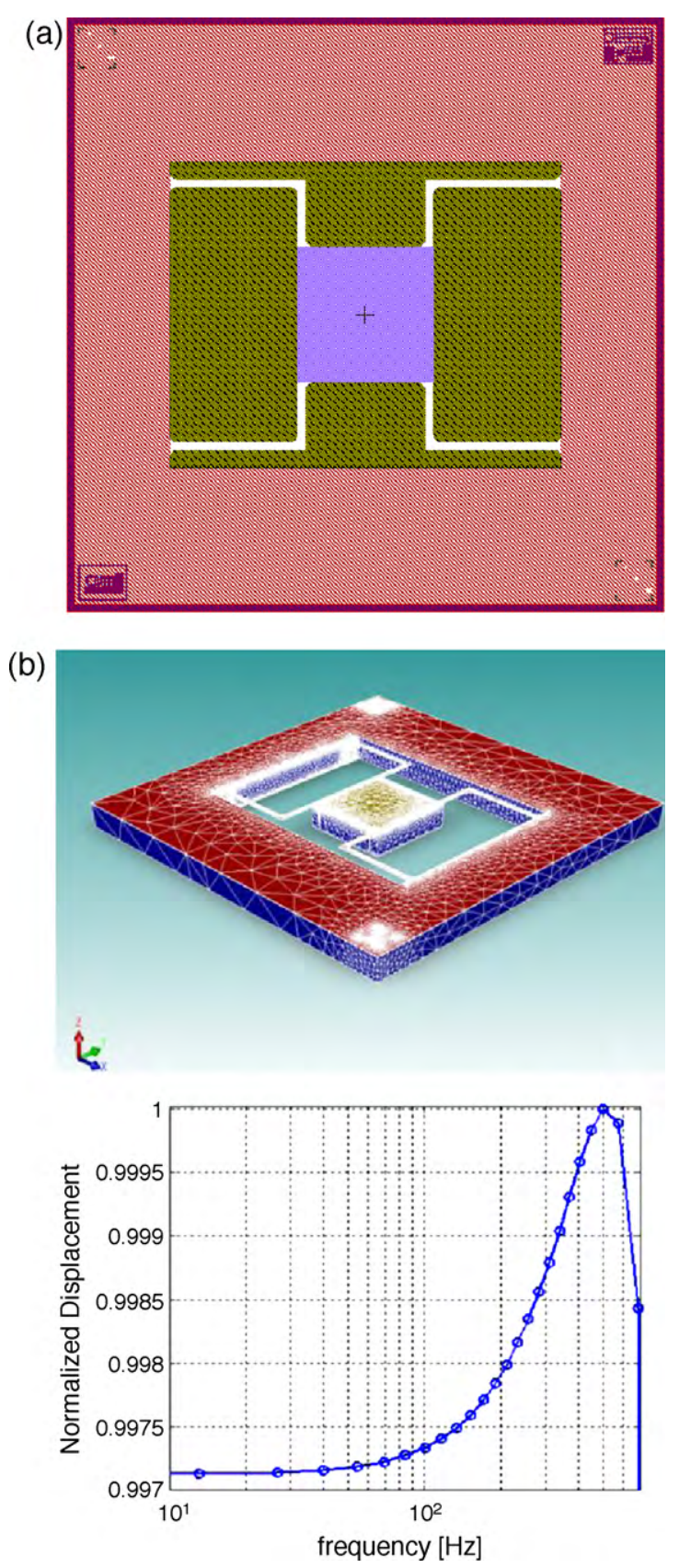

Fig. 3. (a) Layout of the micromachined investigated device based on BESOI technology. (b) Tetrahedrons-based model mesh. (c) Harmonic response along the $z$ axis (normalized to its maximum value).

The equation takes into the account that the spring constant of the flexure is four times the single beam spring constant. $L_{a}$ and $L_{b}$ represent the length of the two beam segments, for the longest leg and for the other one respectively. $A$ and $B$ represent two terms function of the moment of inertia, considering a heterogeneous beam composition and the torsion constant:

$$
\begin{aligned}
& A=E_{\max } I_{n} \\
& B=\frac{E_{\max }}{2\left(1+v_{\max }\right)} J
\end{aligned}
$$

$k_{Z}=\frac{4 F_{Z}}{\delta_{z}}=\frac{48 A^{2}\left(B L_{a}+A L_{b}\right)\left(A L_{a}+B L_{b}\right)}{A^{2} B L_{a}^{5}+4 A^{3} L_{a}^{4} L_{b}+A B^{2} L_{a}^{4} L_{b}+4 A^{2} B L_{a}^{3} L_{b}^{2}+4 A^{2} B L_{a}^{2} L_{b}^{3}+4 A^{3} L_{a} L_{b}^{4}+A B^{2} L_{a} L_{b}^{4}+A^{2} B L_{b}^{5}}$ 


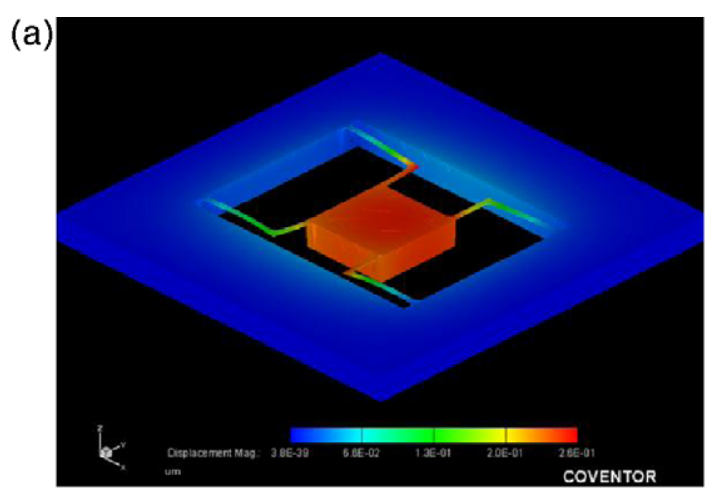

(b)

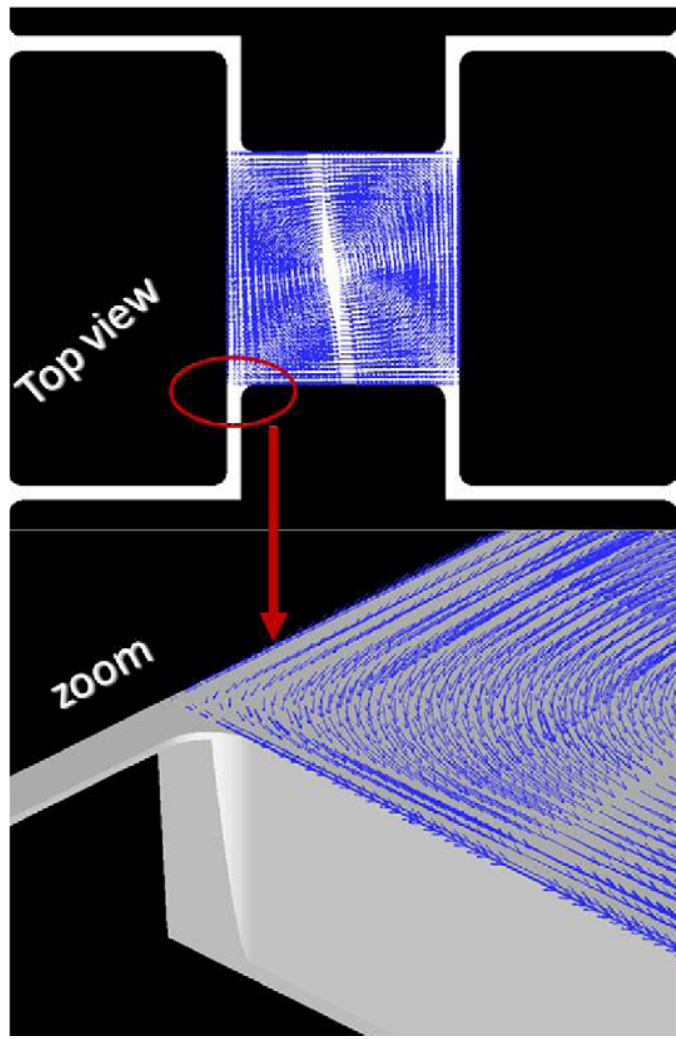

Fig. 4. (a) MemMech analysis: displacement color map in absence of applied load to the plate surface of the microresonator. An exaggeration factor of 400 has been applied just to show the deflection of the structure. (b) MemHenry analysis: eddycurrents distributed onto the micromachined mass. A variable magnetic field having a frequency of $226 \mathrm{~Hz}$ has been imposed through an inductor frontally disposed respect the metal plate. This value represents the excitation frequency necessary to move the passive element at the resonance.
$E_{\max }$ is the maximal value of the stack materials Young's modulus and $v_{\max }$ is the maximal value of the stack materials Poisson's ratio. $I_{n}$ represents the beam inertia's moment evaluated through the heterogeneous beam theory. $J$ is torsion constant for a beam having a rectangular cross section.

A resonance frequency of about $506 \mathrm{~Hz}$ has been computed considering the inertial mass $(M)$ composed by the $450 \mu \mathrm{m}$ of silicon, the buried oxide, the $15 \mu \mathrm{m}$ of crystal silicon, and the oxide layers (Diff, Cont, Metal and Pad).

The springs are composed of $15 \mu \mathrm{m}$ of silicon, and three oxides (Diff, Cont and Pad). The mass of the micromachined device is $3 \mathrm{e}-6 \mathrm{~kg}$ and the spring constant $k_{z}$, estimated through the heterogeneous beam theory, corresponds to about $30.6 \mathrm{~kg} / \mathrm{s}^{2}$. By means of Eq. (5) a first order estimation of the resonance frequency of the structure can be derived.

$f_{r}=\frac{1}{2 \pi} \sqrt{\frac{k_{z}}{M}}$

The resonance agrees with the results obtained by using numerical analysis previously performed through CoventorWare ${ }^{\mathrm{TM}} 2008$. Furthermore, the result is in accordance with the simplified model based on strain energy density along the beam, neglecting the torsion contribution [8].

\section{Experimental results}

The experimental investigation has been performed using a proper designed coil to excite and to sense the device oscillations. In order to increase the signal-to-noise ration in the detection technique, a FeSiB amorphous ferromagnetic microwire [11], prepared by the rotating water spinning method and having the nominal diameter of $100 \mu \mathrm{m}$, has been selected as the core of the coil.

Two superimposed primary windings are used as excitation and probing coils, while two lateral windings, in differential configuration, represent the output pick-up coil. Table 1 summarize the electrical and geometric characteristics of the inductor.

The excitation current corresponds to $19 \mathrm{~mA}_{\mathrm{pp}}$ while the probing bias amplitude is $13 \mathrm{~mA}_{\mathrm{pp}}$. During the experimental campaign a distance between the coil and the microresonator of about $200 \mu \mathrm{m}$ has been maintained.

The excitation signal has been applied to the coil by means of a voltage-current converter amplifier, while a sinusoidal signal has been applied to the probing coil. The output signal from the pickup coil has been analyzed by using two high pass filters and an instrumentation amplifier.

Fig. 5 shows the experimental setup basically composed by a microtranslator, used to move the inductive sensor and to tune its distance as respect the MEMS die which has been fixed on the bottom of the setup, and an acquisition system. A post-processing data elaboration has been performed thorough MATLAB routine.

Table 1

Summary of the electrical and geometrical inductor characteristics.

\begin{tabular}{lllcc}
\hline Specifications & Length $[\mathrm{mm}]$ & Width $[\mathrm{mm}]$ & Resistance $[\Omega]$ & Inductance $[\mathrm{mH}]$ \\
\hline Actuation coil & 14 & 12 & 900 & 220 \\
Probing coil & 14 & 4 & 5.8 & 1.6 \\
Pick-up coil & 1 & 1.5 & 1.6 & 0.0039 \\
\hline
\end{tabular}

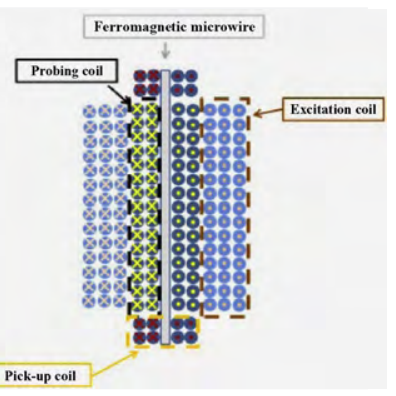



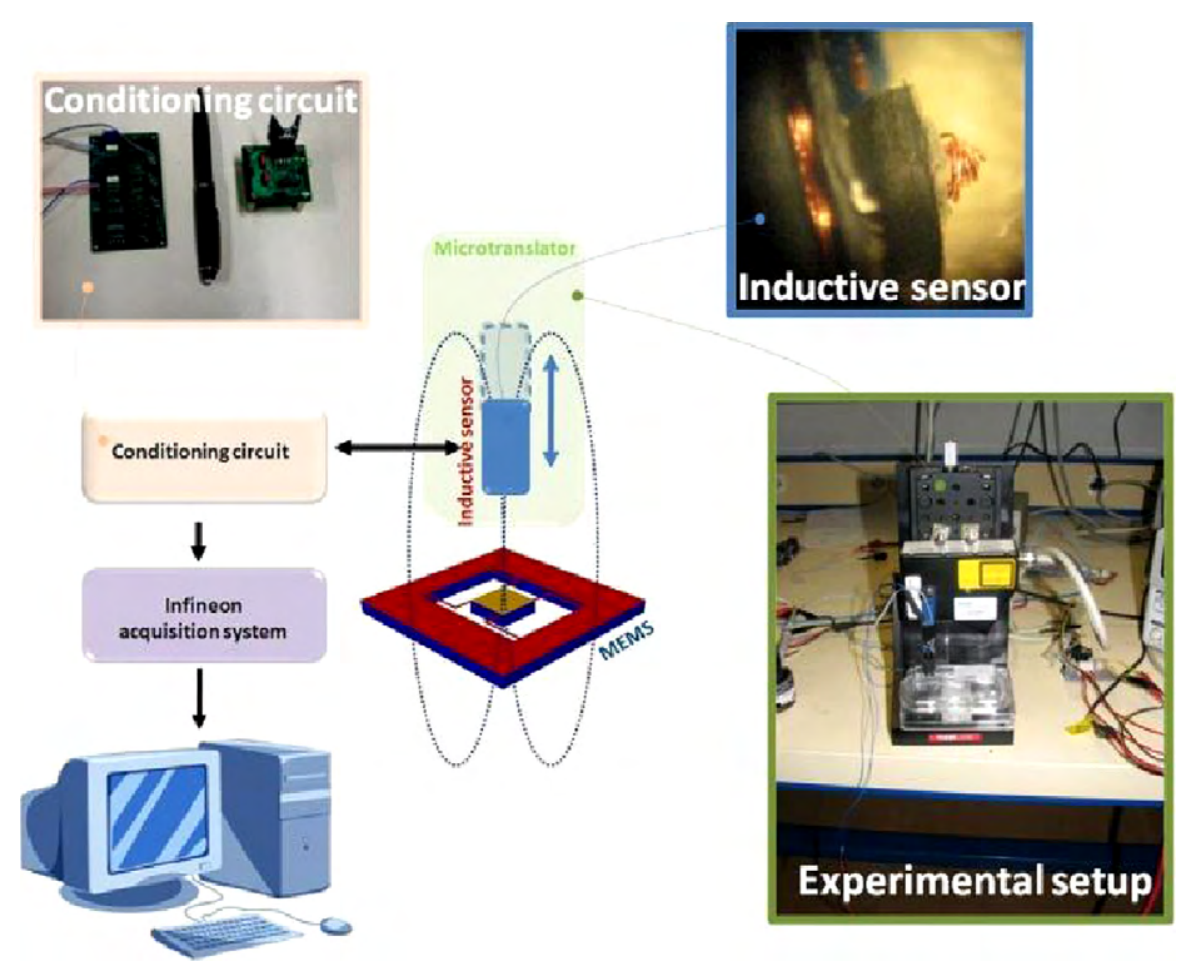

Fig. 5. Schematic diagram of the measurement scheme with details pictures of the individual parts.

(a)
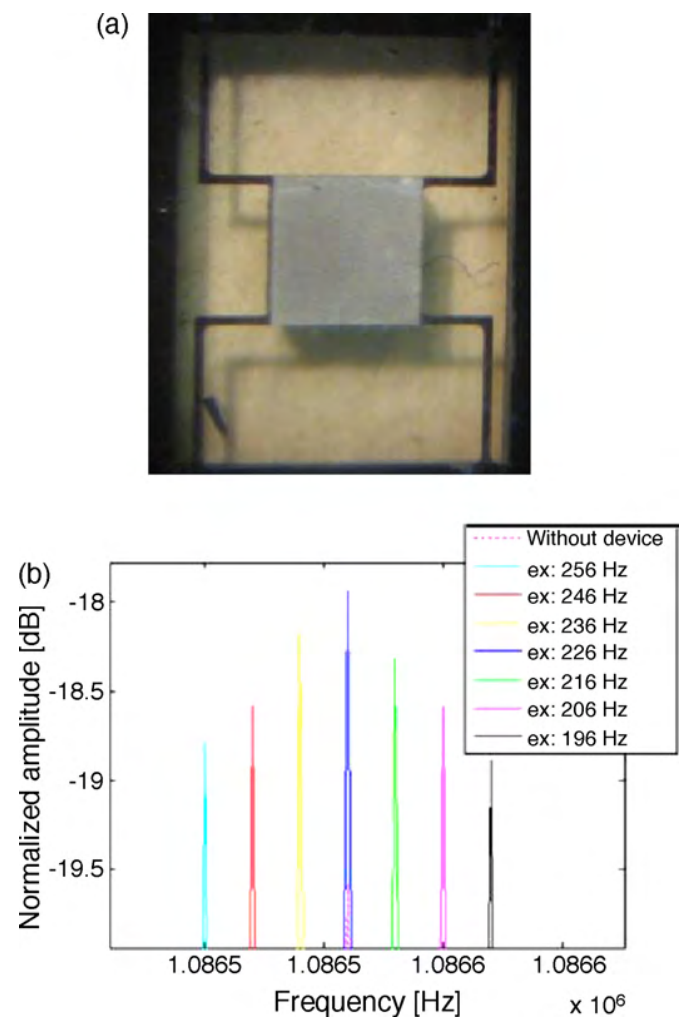

Fig. 6. (a) Microscope picture of the BESOI crab-leg device realized. (b) Validation of the contactless working principle: the central spike represents the contribution at the resonance. An analysis around this value has been conducted by varying the excitation bias frequency. A sinusoidal waveform has been maintained during the experimental campaign.
Fig. 6a shows the realized BESOI device. A sinusoidal signal having an amplitude of $900 \mathrm{mV}_{\mathrm{pp}}$ and a probing bias of $6.5 \mathrm{~V}_{\mathrm{pp}}$ @ $1.087 \mathrm{MHz}$ have been used. The excitation frequency $f_{e}$ around $f_{r} / 2$ has been tuned by using a signal generator, and the output signal has been observed around $f_{r}$, that is the mechanical resonance frequency of the structure. The contactless principle has been validated and a resonant frequency of about $450 \mathrm{~Hz}$ with a quality factor of 1.4 has been experimentally estimated. Fig. $6 \mathrm{~b}$ shows the superposition of the frequency spectra of the magnetic readout signal obtained for different values of the excitation frequency $f_{e}$. It can be observed that the spectra have a resonance envelope centred around the frequency $f_{p}-f_{r}$, that is around the modulated mechanical resonance frequency. An application of the resonator as a mass sensor has also been investigated. To this purpose different quantities of paint have been added on the top surface of the resonator.

The superposition of the frequency spectra of the magnetic readout signal for different values of the excitation frequency $f_{e}$ has been repeated for increasing values of the mass loading. Fig. 7(a) and (b) shows the spectra for the mass loading of respectively 220 and $440 \mu \mathrm{g}$. As it can be observed for each deposited sample a new resonance envelope centered around to a new resonance condition has been detected. From the experimental data, sensitivity of about $0.18 \mathrm{~Hz} / \mu \mathrm{g}$ and a resolution of about $79 \mu \mathrm{g}$ have been experimentally detected. In order to best fit the experimental data with the model (5), a multidimensional unconstrained nonlinear minimization (Nelder-Mead [12]) has been performed. The model used for the optimization process is the following:

$f_{r}=\frac{1}{2 \pi} \sqrt{\frac{a k_{z}}{M}}+b$

where $a$ and $b$ represent the parameters used for the minimization process. Both the terms tend to compensate the discrepancy between the experimental data and the model. As expected, a resonant frequency decrement has been detected as a consequence of different quantities of deposited paint. Fig. 8 shows the evolution of 

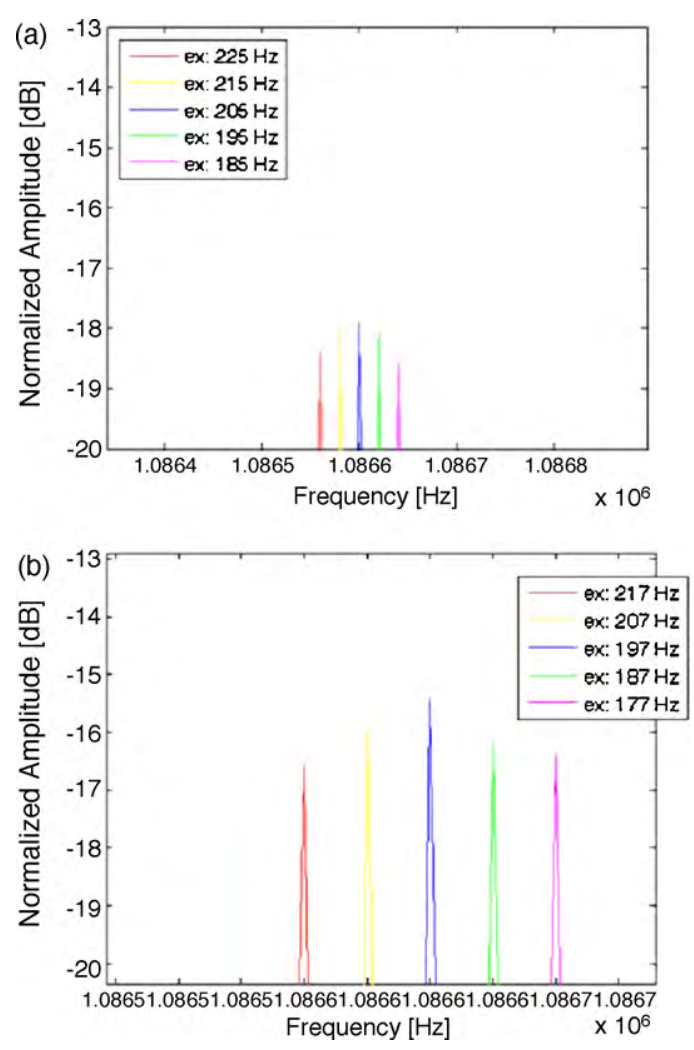

Fig. 7. Analysis around the resonance frequency, different value has been detected as consequence of the masses quantity deposited: (a) $\sim 220 \mu \mathrm{g}$ of paint, (b) $\sim 440 \mu \mathrm{g}$ of paint.

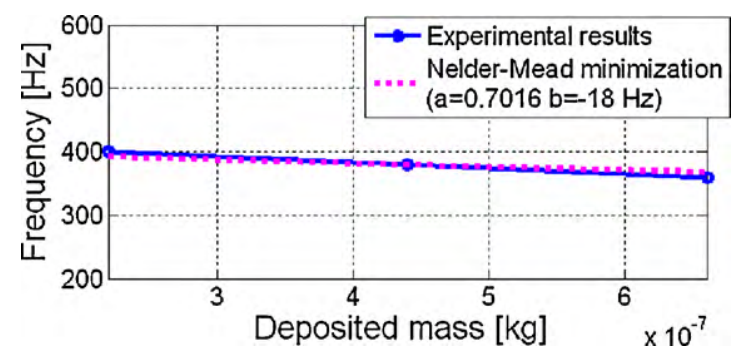

Fig. 8. Experimental results and model performed with a minimization process vs. different quantities of deposited samples. The equation obtained assumes the following expression: $f_{r}=(1 / 2 \pi)\left(\sqrt{\left(0.7016 k_{z} / M\right)}-18 \mathrm{~Hz}\right)$. $M$ represents the mass of the micromachined device and also the contribution of the deposited masses.

the resonance frequency as function of different deposited quantities and compared with the model based on the unconstrained nonlinear minimization.

\section{Conclusions}

In this work the possibility of contactless exciting and detecting mechanical resonances in micromachined resonators has been proved. The principle relies on no specific magnetic property of the resonator except electrical conductivity. The proposed principle exploits the Lorentz force arising from the interaction between an inductively induced eddy-current density and an external timevarying magnetic field. The vibrations of the resonator are detected by exploiting a probing magnetic field which induces on the conductive surface of the resonator an eddy-current density. The magnetic field generated by the induced eddy-current density is modulated by the vibrations of the resonator and it is sensed by an additional pick-up coil.
A four beams crab-leg BESOI-based suspended mass has been designed and fabricated to be used as microresonator. The contactless working principle has been experimentally validated and agreement between the analytical and numerical analysis, realized through CoventorWare ${ }^{\mathrm{TM}}$ 2008, has been found. Furthermore, in order to apply the proposed principle to the sensing of a physical quantity, the device has been studied as contactless mass sensor. An analysis of the resonance frequency variation as consequence of the mass increment has been realized. For each deposited quantity a new resonance frequency has been detected and a resonance envelope around it has been verified. Finally a multidimensional unconstrained nonlinear minimization (Nelder-Mead) has been realized in order to best fit the experimental data with the model. As a future step the optimization of the Q-factor is planned.

The proposed principle can be applied to measure a large variety of physical quantities, which can induce a predictable shift in the oscillation frequency of the resonant structure, such as pressure, temperature or mass loading. Possible applications can be microbalance devices disposed in inaccessible area or biomedical devices for the subcutaneously measurement of biologic entities.

\section{References}

[1] F. Umbach, H. Acker, J. Von Kluge, W. Langheinrich, Contactless measurement of torque, Mechatronics 12 (2002) 1023-1033.

[2] H. Exner, U. Loschner, Contactless laser bending of silicon microstructures, Proc. SPIE 5116 (2003) 383-392.

[3] B. Andò, S. Baglio, M. Baù, V. Ferrari, D. Marioli, E. Sardini, N. Savalli, M. Serpelloni, C. Trigona, Development of contactless resonant MEMS force sensors in SOI technology, in: Proceeding of Eurosensors 2008, Dresden, Germany, September 7-10, 2008.

[4] Y. Ju, K. Inoue, M. Saka, H. Abe, Contactless measurement of electrical conductivity of semiconductor wafers using the reflection of millimeter waves, Appl. Phys. Lett. 81 (19) (2002) 3585-3587.

[5] M. Baù, V. Ferrari, D. Marioli, E. Sardini, M. Serpelloni, A. Taroni, Contactless electromagnetic excitation of resonant sensors made of conductive miniaturized microstructures, Sens. Actuators A 148 (2008) 44-50.

[6] M. Baù, V. Ferrari, D. Marioli, E. Sardini, M. Serpelloni, A. Taroni, Contactless excitation and readout of passive sensing elements made by miniaturized mechanical resonators, in: IEEE Sensors, October 28-31, 2007, pp. 36-39.

[7] C. De Angelis, V. Ferrari, D. Marioli, E. Sardini, M. Serpelloni, A. Taroni, Magnetically induced oscillations on a conductive cantilever for resonant microsensors, Sens. Actuators A 135 (2007) 197-202.

[8] B. Andò, S. Baglio, M. Baù, V. Ferrari, E. Sardini, N. Savalli, M. Serpelloni, C. Trigona, Contactless electromagnetic interrogation of a MEMS-based microresonator used as passive sensing element, in: Proceeding of Transducers, 2009, pp. 1429-1432.

[9] B. Andò, et al., Numerical and experimental investigation on contactless resonant sensors, Sens. Actuators A: Phys. 160 (2) (2010) 329-335.

[10] G.K. Fedder, Simulations of microelectromechanical systems, PhD Thesis, Department of Electrical Engineering and Computer Science, University of California at Berkeley, Berkeley, California, 1994

[11] B. Andò, S. Baglio, A.R. Bulsara, C. Trigona, Design and characterization of a microwire-fluxgate, Sens. Actuators A: Phys. 151 (2) (2009) 145-153.

[12] J.A. Nelder, R. Mead, A simplex method for function minimization, Comput. J. 7 (1965) 308-313.

\section{Biographies}

Bruno Andò received MS in Electronic Engineering and PhD in Electrical Engineering from the Università di Catania, Italy, in 1994 and 1999, respectively. From 1999 to 2001, he worked as a researcher with the Electrical and Electronic Measurement Group of the University of Catania - DEES. In 2002, he became an assistant professor with the same staff. His main research interests are sensors design and optimization including advanced multi-sensors architecture for visually impaired people, characterization of new materials for sensors, nonlinear techniques for signal processing with particular interest in stochastic resonance and dithering applications; characterization and conditioning; distributed measurement systems. During his activity Dr. Andò has co-authored several scientific papers, presented in international conferences and published in international journals and books.

S. Baglio received the "Laurea" and $\mathrm{PhD}$ degrees from the University of Catania Catania, Italy, in 1990 and 1994, respectively. Since 1996 he has been with the Dipartimento di Ingegneria Elettrica Elettronica e dei Sistemi, University of Catania where he is currently Associate Professor. Prof. Baglio teaches courses in "measurement theory," "electronic instrumentations," and "integrated microsensors". He is 
a co-author of more than 250 scientific publications, which include books, chapters in books, papers in international journals and proceedings of international conferences. He is also co-holder of several U.S. patents. Prof. Baglio is Senior Member of the IEEE, he has served as Associate Editor for the IEEE Transactions on Circuits and Systems-I, as Distinguished Lecturer for the IEEE Circuits and Systems Society and is currently Associate Editor of the IEEE Transaction on Instrumentation and Measurements. His research interests are mainly focused on microsensors and nanosensors, hysteretic materials for sensors, nonlinear dynamics for transducers.

M. Baù was born in 1981 in Castiglione delle Stiviere, Italy. He received the Laurea degree cum laude in Electronic Engineering and the Research Doctorate degree in Electronic Instrumentation from the University of Brescia in 2005 and 2009, respectively. Since 2009 he has been Research Assistant in Electronics with the Department of Information Engineering (ex Department of Electronics for Automation), University of Brescia. His main research activity deals with the design of electronics circuit for sensor interface and the development of techniques for the contactless activation and detection of resonant sensors with particular regard to MEMS resonators.

V. Ferrari was born in Milan, Italy, in 1962. In 1988, he obtained the Laurea degree cum laude in Physics at the University of Milan. In 1993 he received the Research Doctorate degree in Electronic Instrumentation at the University of Brescia. He had been an assistant professor and an associate professor at the Faculty of Engineering of the University of Brescia until 2001 and 2006, respectively. Since 2006 he has been a full professor of Electronics. His research activity is in the field of sensors and the related signal-conditioning electronics. Particular topics of interest are acousticwave piezoelectric sensors, microresonant sensors and MEMS, autonomous sensors and power scavenging, oscillators for resonant sensors and frequency-output interface circuits. He is involved in national and international research programmes, and in projects in cooperation with industries.

E. Sardini was born in Commessaggio, Mantova, Italy in 1958. He graduated in Electronic Engineering at the Politecnico of Milan, Italy, in 1983. Since 1984 he has joined the department of Information Engineering of the University of Brescia, Italy. Since 2006 he has been a Full Professor in Electrical and Electronics Measurements. His research activity is in the field of sensors and electronic instrumentation; in particular he developed the conditioning electronics mainly for capacitive and inductive sensors, microprocessor based instrumentation, thick film sensors, instrumentation for noise measurement and for low frequency acceleration measurements. Recently the research interest has been addressed to autonomous sensor for biomedical applications. He is also a coordinator of the technology for Health $\mathrm{PhD}$.

N. Savalli received master degree and PhD from the University of Catania in 1999 and in 2003, respectively. He is currently a Research Associate at the Dipartimento di Ingegneria Elettrica, Elettronica e dei Sistemi (DIEES), University of Catania, Italy. Since 2003 he has been lecturer of Applied Electronic Measurements and Electrical Measurements at DIEES, University of Catania. He is co-author of more than 90 scientific publications including international journals, international conferences and national conferences. His main research interests include soft computing methodologies for instrumentation and measuring systems, smart sensors, material characterization, device applications of non linear dynamics, microsensors and microsystems in standard and dedicated technologies, photonic band gap materials and optical filters.

M. Serpelloni was born in Brescia, Italy, in 1979. He received the Laurea degree (summa cum laude) in industrial management engineering and the Research Doctorate degree in electronic instrumentation from the University of Brescia, Brescia, in 2003 and 2007, respectively. He is currently a Research Assistant of electrical and electronic measurements with the Department of Information Engineering, University of Brescia. He has worked on several projects relating to the design, modeling, and fabrication of measurement systems for industrial applications. His research interests include contactless transmissions between sensors and electronics, contactless activation for resonant sensors, and signal processing for microelectromechanical systems.

Carlo Trigona was born in Siracusa, Italy, in 1981. He received his MS degree in Automation Engineering and Control of Complex System and the PhD degree in Electronic, Automation and Control of Complex System from University of Catania, Catania, Italy in 2006 and 2009 respectively. He is a co-author of more than 40 scientific publications, which include chapters in books, papers in international journals and proceedings of international conferences. His research interests include microsystems and microsensors, fluxgate magnetometers, analog and digital electronic circuit designs. 